\title{
Importance of viscosity contrast for the motion of erythrocytes in microcapillaries
}

\author{
Anil K. Dasanna ${ }^{1}$, Johannes Mauer ${ }^{1}$, Gerhard Gompper ${ }^{1}$ and Dmitry A. \\ Fedosov ${ }^{1, *}$ \\ ${ }^{1}$ Theoretical Physics of Living Matter, Institute of Biological Information Processing \\ and Institute for Advanced Simulation, Forschungszentrum Jülich, 52425 Jülich, \\ Germany \\ Correspondence*: \\ Dmitry A. Fedosov \\ d.fedosov@fz-juelich.de
}

Keywords: red blood cell, channel flow, cell shape, cell dynamics, cell deformation, mesoscopic simulation

\section{INTRODUCTION}

The dynamics and deformation of red blood cells (RBCs) in microcirculation affect the flow resistance and transport properties of whole blood. One of the key properties that can alter RBC dynamics in flow is the contrast $\lambda$ (or ratio) of viscosities between RBC cytosol and blood plasma. Here, we study the dependence of RBC shape and dynamics on the viscosity contrast in tube flow, using mesoscopic hydrodynamics simulations. State diagrams of different RBC dynamical states, including tumbling cells, parachutes, and tank-treading slippers, are constructed for various viscosity contrasts and wide ranges of flow rates and tube diameters (or RBC confinements). Despite similarities in the classification of RBC behavior for different viscosity contrasts, there are notable differences in the corresponding state diagrams. In particular, the region of parachutes is significantly larger for $\lambda=1$ in comparison to $\lambda=5$. Furthermore, the viscosity contrast strongly affects the tumbling-to-slipper transition, thus modifying the regions of occurrence of these states as a function of flow rate and RBC confinement. Also, an increase in cytosol viscosity leads to a reduction in membrane tension induced by flow stresses. Physical mechanisms that determine these differences in RBC dynamical states as a function of $\lambda$ are discussed.

Microvascular blood flow is essential for the homeostasis of organism tissues, as it transports nutrients and waste products and mediates various physiological processes. This research field has received enormous attention directed at understanding complex microvascular transport and regulation [1, 2, 3, 4, 5]. Blood is a liquid tissue whose major cellular component is erythrocytes or red blood cells (RBCs) which constitute about $45 \%$ of blood volume. A healthy RBC has a biconcave shape with a diameter of $6-8 \mu \mathrm{m}$ and thickness of $2 \mu \mathrm{m}[6]$. The RBC membrane consists of a lipid bilayer and spectrin network (cytoskeleton) attached to the inside of the bilayer [7]. These structures supply cell deformability and durability, as RBCs have to frequently pass capillaries with a diameter comparable to the RBC size. The ability of RBCs to deform is vital for microvascular perfusion, as an increased membrane rigidity is generally associated with pathological conditions [8, 9] such as sickle-cell anemia [10] and malaria [11, 12]. 
bioRxiv preprint doi: https://doi.org/10.1101/2021.02.11.430779; this version posted February 11, 2021. The copyright holder for this preprint (which was not certified by peer review) is the author/funder, who has granted bioRxiv a license to display the preprint in perpetuity. It is made available under aCC-BY-NC 4.0 International license.

One of the important steps toward understanding microvascular blood flow is a detailed description of $\mathrm{RBC}$ behavior in microcapillaries. Early experiments [13, 14, 15] have shown that RBCs passing through small vessels either deform into cup-like parachute shapes at the vessel center or assume elongated slipper shapes at an off-center position. A number of more recent microfluidic experiments [16, 17, 18, 19, 20] have systematically studied and confirmed these observations and suggested a connection between RBC elasticity and its shape in flow. From the physics point of view, it is interesting to understand how such shapes develop and which cell and flow properties determine their stability. First simple axisymmetric models of RBCs flowing in microvessels [21] have demonstrated the ability of RBCs to attain parachute and bullet-like (in very narrow vessels) shapes due to the stresses exerted by fluid flow. Two dimensional (2D) simulations of fluid vesicles mimicking RBCs have shown that cell behavior in microcapillary flow is quite complex [22, 23, 24, 25, 26, 27]. In addition to the parachute and slipper shapes, snaking dynamics (a periodic cell swinging around the tube center) at low flow rates and a region of co-existing parachutes and slippers at high flow rates were reported [24, 25]. These 2D simulations have also demonstrated that the transition between parachute and slipper shapes can be triggered by changes in flow rate or RBC membrane elasticity. This transition can be characterized by the distance between the cell's center-of-mass and the channel center, which has been shown to have a similar behavior as a pitchfork bifurcation [23]. Nevertheless, it is still not fully clear why the parachute-to-slipper transition takes place.

Three dimensional (3D) simulations of RBCs flowing in microchannels [28, 29, 30, 31, 32, 33, 34] have confirmed the existence of stable slippers in 3D. Despite some similarities between the results obtained from 2D and 3D simulations, RBC dynamics in microchannels is inherently three dimensional, so that the results from 2D simulations are at most qualitative. For instance, 3D simulations have shown the existence of a dynamic state of RBC tumbling at a radial position away from the tube center [29, 34]. In fact, the transition from tumbling to slipper state with increasing flow rate is reminiscent of the well-known tumbling-to-tank-treading transition of RBCs in simple shear flow [35, 36, 37, 38]. Furthermore, recent experiments on RBCs in flow within square microchannels have found a tumbling trilobe state at large flow rates and low confinements [34]. Such trilobe dynamics has so far only been reproduced in simulations of RBCs in simple shear flow, and occurs at large shear rates and for large enough viscosity contrasts $\lambda$ defined as the ratio between viscosities of RBC cytosol and suspending medium [39, 40], with $\lambda \gtrsim 3.5$.

Most of the current simulation studies assume for simplicity the viscosity contrast of unity, even though the average physiological value of $\lambda$ is about five [41, 42]. The viscosity contrast is an important parameter that significantly affects RBC behavior in simple shear flow [39, 40, 43, 44, 45]. However, it remains unclear whether the viscosity contrast is important for RBC dynamics in microcapillary flow. Therefore, we focus on the effect of $\lambda$ on RBC dynamical states in tube flow. Several state diagrams of RBC dynamics, including snaking, tumbling, tank-treading slipper, and parachute, are presented for different viscosity contrasts, tube diameters, and flow rates. Even though the dynamical states are similar for $\lambda=1$ and $\lambda=5$, there are differences in flow conditions at which they appear. In particular, the region of tumbling dynamics for $\lambda=5$ expands toward larger flow rates in comparison to $\lambda=1$, since an increased dissipation inside the cell suppresses membrane tank-treading in favor of tumbling motion. Furthermore, the region of parachute shapes is larger for $\lambda=1$ than that for $\lambda=5$. A larger viscosity inside the $\mathrm{RBC}$ also leads to a decrease in membrane tension for the same flow conditions. Physical mechanisms that determine these differences in dynamical state diagrams for various viscosity contrasts are discussed. 


\section{MODELS \& METHODS}

\subsection{Red blood cell model}

72

A RBC is modeled as a triangulated surface with $N_{\mathrm{v}}=3000$ vertices, $N_{\mathrm{e}}$ edges, and $N_{\mathrm{f}}$ triangular faces [46, 28, 47, 48]. The total potential energy of the system is given by

$$
V=V_{\text {in-plane }}+V_{\text {bend }}+V_{\text {area }}+V_{\text {vol }}
$$

The term $V_{\text {in-plane }}$ represents an in-plane elastic energy as [47, 48]

$$
V_{\text {in-plane }}=\sum_{i=1}^{N_{\mathrm{e}}} \frac{k_{B} T \ell_{\mathrm{m}}\left(3 x_{i}^{2}-2 x_{i}^{3}\right)}{4 p\left(1-x_{i}\right)}+\sum_{i=1}^{N_{\mathrm{e}}} \frac{k_{\mathrm{p}}}{\ell_{i}},
$$

where the first term is an attractive worm-like chain potential and the second term is a repulsive potential with a strength coefficient $k_{\mathrm{p}}$. In the attractive potential, $p$ is the persistence length, $\ell_{i}$ is the extension of edge $i, \ell_{\mathrm{m}}$ is the maximum edge extension, and $x_{i}=\ell_{i} / \ell_{\mathrm{m}}$.

The second term in Eq. (1) corresponds to bending resistance of the membrane,

$$
V_{\mathrm{bend}}=\sum_{i=1}^{N_{\mathrm{e}}} \kappa_{\mathrm{b}}\left(1-\cos \left(\theta_{i}-\theta_{0}\right)\right),
$$

where $\kappa_{\mathrm{b}}$ is the bending coefficient, $\theta$ is the angle between two neighboring faces, and $\theta_{0}$ is the spontaneous angle. The last two terms in Eq. (1), $V_{\text {area }}$ and $V_{\text {vol }}$, represent surface area and volume constraints given by

$$
\begin{aligned}
V_{\text {area }} & =\frac{k_{\mathrm{a}}\left(A-A_{0}\right)^{2}}{2 A_{0}}+\sum_{i=1}^{N_{\mathrm{f}}} \frac{k_{\mathrm{d}}\left(A_{i}-A_{i}^{0}\right)^{2}}{2 A_{i}^{0}}, \\
V_{\text {vol }} & =\frac{k_{\mathrm{v}}\left(V-V_{0}\right)^{2}}{2 V_{0}}
\end{aligned}
$$

where $k_{\mathrm{a}}, k_{\mathrm{d}}$, and $k_{\mathrm{v}}$ are local area, total surface area and volume constraint coefficients, respectively. $A_{i}^{0}$, $A_{0}$ and $V_{0}$ are local area of individual faces, total surface area and total volume of the RBC, respectively.

\subsection{Modeling hydrodynamic flow}

The fluid is modeled by the smoothed dissipative particle dynamics (SDPD) method which is a Lagrangian discretization of Navier-Stokes equations [49, 50]. The SDPD fluid consists of $N$ fluid particles which interact through conservative, dissipative and random forces. The solvent inside the RBC (cytosol) is separated from outside fluid (plasma) by the membrane. The number density of fluid particles is set to $n=12$ (per unit volume in model units) for both cytosol and plasma. Solid walls are modeled by frozen SDPD particles. Fluid-membrane interactions have two contributions: $(i)$ fluid particles bounce back from the membrane surface and $(i i)$ the dissipation force coefficient between fluid particles and membrane vertices is set such that no-slip boundary conditions are attained. Fluid particles are also reflected back at the solid wall. In addition, an adaptive shear force is added to fluid particles near the wall to ensure no-slip boundary conditions [51]. 


\subsection{Simulation setup and parameters}

Poiseuille flow with a single RBC suspended in a viscous fluid inside a cylindrical tube of length $L=50 \mu \mathrm{m}$ is simulated. The tube axis is aligned with the flow direction along the $\mathrm{x}$ axis. Diameter of the tube $D=2 R$ determines RBC confinement as $\chi=D_{r} / D$, where $D_{r}=\sqrt{A_{0} / \pi}$ is the effective RBC diameter. To generate flow, a force $f$ is applied on every solvent particle, representing a pressure gradient $\Delta P / L=f \cdot n$ with the pressure drop $\Delta P$ along the tube length.

In simulations, cell properties correspond to average characteristics of a healthy RBC with a membrane area $A_{0}=133 \mu \mathrm{m}^{2}$, cell volume $V_{0}=93 \mu \mathrm{m}^{3}$, shear modulus $\mu=4.8 \mu \mathrm{N} / \mathrm{m}$, and bending rigidity $\kappa=70 k_{\mathrm{B}} T=3 \times 10^{-19} \mathrm{~J}$ [6, 52, 53, 54]. This leads to $D_{r}=6.5 \mu \mathrm{m}$ ( $D_{r}=6.5 \mathrm{in}$ model units) and a RBC reduced volume of $V^{*}=6 V_{0} /\left(\pi D_{r}^{3}\right) \approx 0.64$. Note that the stress-free shape of a RBC elastic network [Eq. (2)] is assumed to be an oblate spheroid with a reduced volume of 0.96 . Furthermore, the energy unit $k_{\mathrm{B}} T$ is selected to be $k_{\mathrm{B}} T=0.2$ in simulations, corresponding to a physiological temperature of $37^{\circ} \mathrm{C}$.

To characterize different flow conditions, several non-dimensional parameters are employed

(i) Reynolds number $R e=\rho \overline{\dot{\gamma}} D_{r}^{2} / \eta$ is the ratio of inertial and viscous forces, where $\rho$ is the mass density, $\overline{\dot{\gamma}}=\bar{v} / D=D f n /(32 \eta)$ is the average (or pseudo) shear rate, and $\eta$ is the external fluid viscosity. In all simulations, $R e \leq 0.3$.

(ii) $\lambda=\eta_{i} / \eta_{o}$ is the viscosity contrast between internal (cytosol) and external (plasma) fluids. The average value of $\lambda$ under physiological conditions is $\lambda=5$ [41, 42].

(iii) $\dot{\gamma}^{*}=\overline{\dot{\gamma}} \cdot \tau$ is the dimensionless shear rate that characterizes flow strength. $\tau$ is the RBC relaxation time given by $\tau=\eta D_{r} / \mu$.

To keep Reynolds number low enough (i.e., $R e \leq 0.3$ ), in most simulations $\dot{\gamma}^{*}$ is controlled by varying $\eta$ instead of changing the flow rate for a fixed viscosity.

\subsection{Dynamical characteristics and membrane tension}

To analyze dynamical properties of a flowing RBC, the gyration tensor

$$
T_{i j}=\frac{1}{N} \sum_{n=1}^{N}\left(r_{n, i}-r_{c, i}\right) \cdot\left(r_{n, j}-r_{c, j}\right)
$$

115 is employed, where $i$ and $j$ denote $x, y$, or $z, \mathbf{r}_{n}$ is the position of membrane vertex $n$, and $\mathbf{r}_{c}$ is the center 116 of mass of the RBC. Then, the eigenvalues $\xi_{i}$ of the gyration tensor $T_{i j}$ characterize RBC deformation. 117 The eigenvector that corresponds to the smallest eigenvalue is used to define the orientational axis of the 118 cell. Orientation angle $\theta_{1}$ of the RBC is defined as the angle between its orientational axis and the flow 119 direction. The eigenvalues are also used to compute cell asphericity $\mathcal{O}$, which characterizes its deviation 120 from a spherical shape

$$
\mathcal{O}=\left[\left(\xi_{1}-\xi_{2}\right)^{2}+\left(\xi_{2}-\xi_{3}\right)^{2}+\left(\xi_{3}-\xi_{1}\right)^{2}\right] /\left(2 R_{g}^{4}\right)
$$

121 where $R_{g}^{2}=\xi_{1}+\xi_{2}+\xi_{3}$. 
To calculate local membrane tension $G_{i}$ at vertex $i$, virial stress is used as

$$
G_{i}=-\frac{a_{i}}{2} \sum_{j(i)} \mathbf{r}_{\mathrm{i}, \mathrm{j}} \cdot \mathbf{F}_{\mathrm{i}, \mathrm{j}}
$$

where $a_{i}$ is the vertex area computed as one third of a sum of all face areas adjacent to vertex $i, j(i)$ represents all neighboring vertices connected to $i$ by an edge, and $\mathbf{r}_{i, j}$ and $\mathbf{F}_{\mathrm{i}, \mathrm{j}}$ are position and force vectors at the edge $(i, j)$, respectively. Note that the in-plane elastic energy, bending potential, surface area, and volume constraints can contribute to the membrane tension.

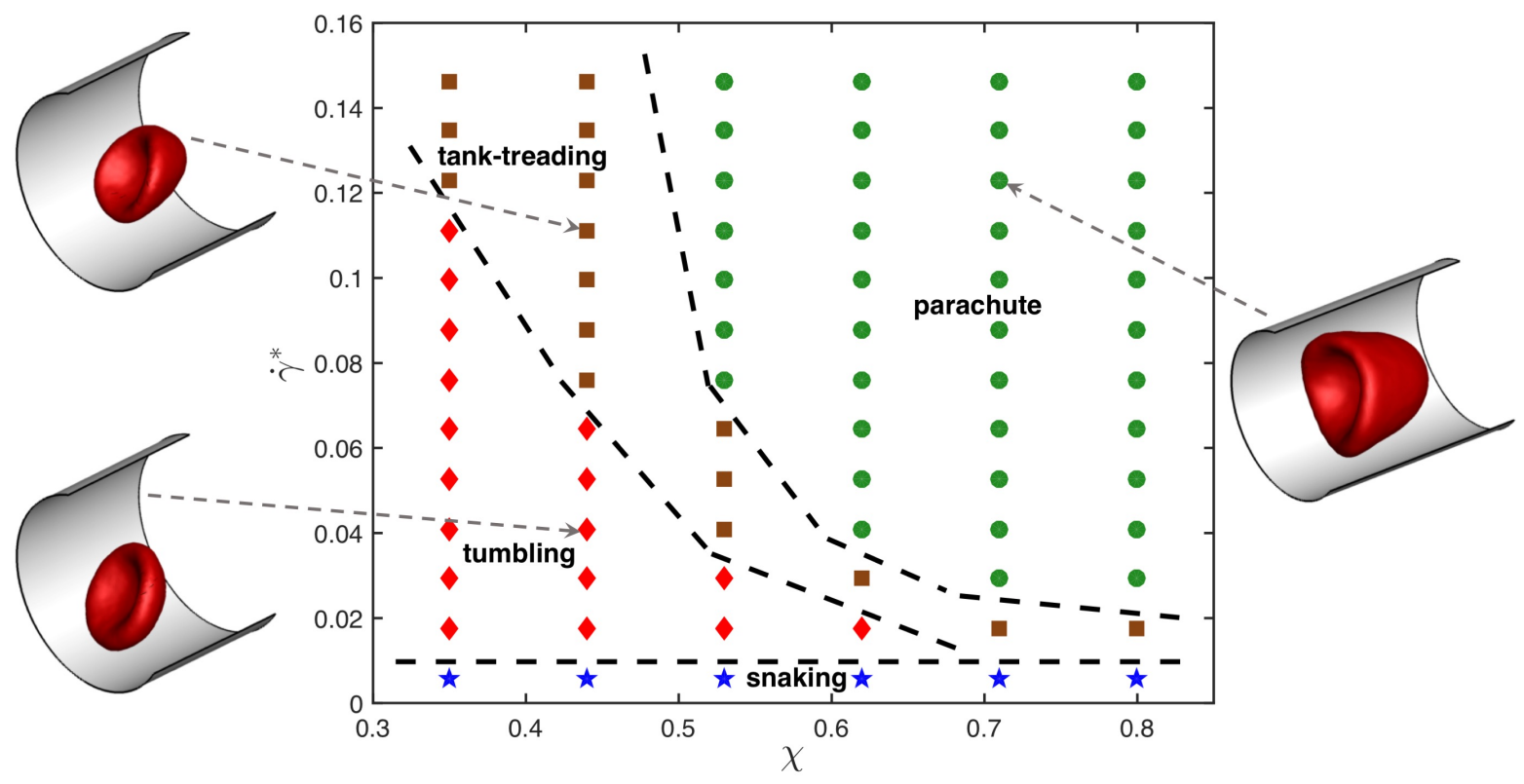

Figure 1. State diagram for $\lambda=5$ showing different dynamical states of the RBC for various confinement ratios $\chi$ and non-dimensional shear rates $\dot{\gamma}^{*}$. The states include snaking (blue stars), tumbling (red diamonds), tank-treading (brown squares), and parachute (green circles). Dashed lines separating regions with different states are drawn for visual guidance. Representative snapshots for tumbling, tank-treading, and parachute states are also displayed.

\section{RESULTS}

In microcapillary flow, RBCs are known to exhibit different dynamical states, including snaking, tumbling, tank-treading, and parachute [15, 17, 20, 24, 25, 29, 34]. Snaking is characterized by a periodic swinging in $\mathrm{RBC}$ orientation around the tube axis [24, 25, 29]. Tumbling is an off-axis rigid-body-like rotation, similar to RBC tumbling in simple shear flow [37, 40, 55]. Tank-treading is represented by membrane rotation with a nearly fixed cell orientation, which also occurs in simple shear flow at low enough $\lambda[$ [37, 40, 56]. The tank-reading state of a RBC in microcapillary flow is also often referred to as slipper. Finally, parachute is a stable stomatocyte-like RBC deformation in the tube center. These dynamical states depend on RBC mechanical properties (e.g., shear modulus, bending rigidity, viscosity contrast), cell confinement, and the flow rate. Here, we primarily focus on how the viscosity contrast affects these dynamical states for a wide range of RBC confinements and flow rates. 


\subsection{Dynamic state diagram}

Figure 1 presents dynamic state diagram for the viscosity contrast $\lambda=5$ and different $\chi$ and $\dot{\gamma}^{*}$ values. The representative snapshots of tumbling, tank-treading, and parachute states are also displayed (see Movies S1-S3). The snaking state exhibits minimal deformation and appears at very low shear rates $\dot{\gamma}^{*} \lesssim 0.01$ for all confinements $\chi$. The tumbling state occurs for small confinements and moderate shear rates. As the shear rate increases, a tumbling RBC transits into a tank-treading state. The critical shear rate, at which the tumbling-to-tank-treading transition takes place, depends on $\chi$ and increases with increasing confinement. For large enough confinements and shear rates, the RBC adopts a parachute shape which exhibits least dynamics out all observed states.
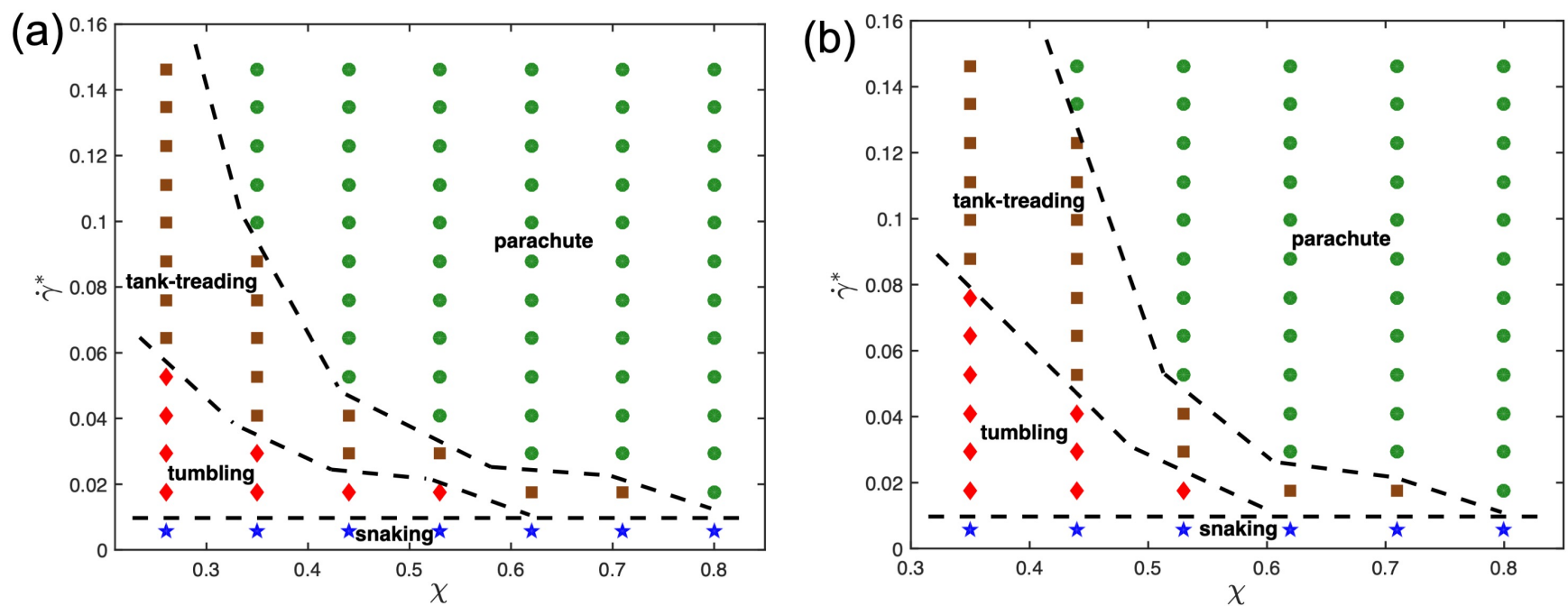

Figure 2. State diagrams for viscosity contrasts (a) $\lambda=1$ and (b) $\lambda=3$ with snaking (blue stars), tumbling (red diamonds), tank-treading (brown squares), and parachute (green circles) states. Dashed lines separating regions with different states are drawn for visual guidance.

To understand the effect of viscosity contrast on dynamical states of the RBC in microcapillary flow, the state diagrams for $\lambda=1$ and $\lambda=3$ are shown for comparison in Fig. 2. As the viscosity contrast is decreased from $\lambda=5$ to $\lambda=1$, the parachute region widens toward smaller confinement values. This is a surprising result considering the fact that an increase in viscosity contrast suppresses tank-treading in simple shear flow [39, 40], which will be discussed later. The tumbling-to-tank-treading transition shifts toward larger shear rates as the viscosity contrast is increased from $\lambda=1$ to $\lambda=5$. This result is consistent with our expectations that an increase in internal viscosity leads to increased fluid stresses inside the RBC, suppressing membrane tank-treading. A similar observation has also been made in the context of adhered malaria-infected RBCs (iRBCs) under flow, such that an increase in viscosity contrast suppresses iRBC crawling at the surface and results in iRBC flipping or its complete detachment [57]. Note that the snaking state remains nearly unchanged by the viscosity contrast.

\subsection{Dynamical characteristics}

To examine differences in dynamical characteristics of RBCs with a change in viscosity contrast, multiple dynamical measures which uniquely characterize each state are computed. Figure 3 presents time evolution of the orientation angle $\theta_{1}$ and asphericity $\mathcal{O}$ for two different flow conditions $\left(\chi=0.35 \& \dot{\gamma}^{*}=0.076\right.$; $\left.\chi=0.44 \& \dot{\gamma}^{*}=0.1\right)$ and viscosity contrasts $\lambda=1$ and $\lambda=5$. For the case with $\chi=0.35$ and 

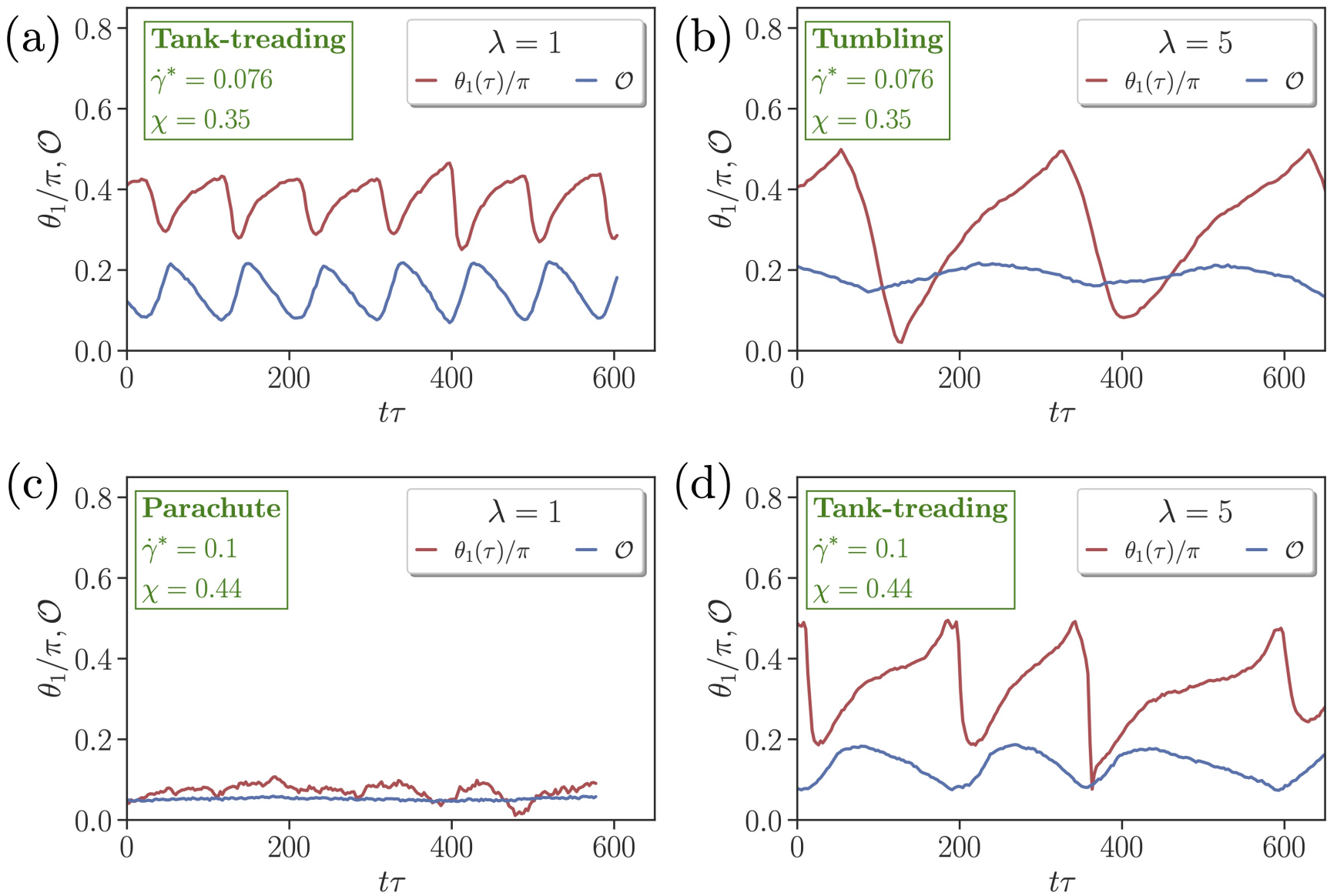

Figure 3. Comparison of time-dependent cell orientation $\theta_{1}$ and asphericity $\mathcal{O}$ for $[$ (a) \& (c) $] \lambda=1$ and [(b) \& (d)] $\lambda=5$. Two flow conditions are selected, including [(a) \& (b)] $\chi=0.35$ and $\dot{\gamma}^{*}=0.076$, and [(c) \& (d)] $\chi=0.44$ and $\dot{\gamma}^{*}=0.1$. Both (a) and (d) represent tank-treading states, whereas (b) corresponds to a tumbling state and (c) to a parachute state.

$\dot{\gamma}^{*}=0.076$ in Fig. 3(a) \& (b), the RBC tank-treads for $\lambda=1$, whereas it tumbles for $\lambda=5$. In an idealized tank-treading state with only membrane rotation and without cell deformation, both $\theta_{1}$ and $\mathcal{O}$ should remain constant. However, a moderate periodic deformation and oscillatory orientation swinging is observed in Fig. 3(a) for $\lambda=1$. For the tumbling state in Fig. 3(b) with $\lambda=5$, membrane deformation is significantly reduced, and the orientation angle spans a much wider range, indicating whole-cell flipping. For the case with $\chi=0.44$ and $\dot{\gamma}^{*}=0.1$ in Fig. 3 (c) \& (d), $\lambda=1$ results in a parachute state with nearly constant $\theta_{1}$ and $\mathcal{O}$, while $\lambda=5$ leads to a tank-treading state with variations in $\theta_{1}$ and $\mathcal{O}$ resembling those in Fig. 3.a). Interestingly, the frequency of the variations in $\theta_{1}$ and $\mathcal{O}$ for $\lambda=5$ in Fig. 3. (d) is significantly smaller than that $\lambda=1$ in Fig. 3(a), even though the shear rate is larger for $\lambda=5$. This means that an increased internal viscosity slows down dynamic deformations of the RBC in microcapillary flow.

\subsection{Membrane tension}

It is interesting to take a look at the effect of viscosity contrast on local membrane tension, as it might be important for the activation of mechano-sensitive channels within the membrane [58, 59]. Figure 4(a) shows the distribution of local tension $G$ for a parachute shape normalized by the shear modulus $\mu$. The local tension $G$ is calculated using Eq. (7), where all contributions from model potentials in Eq. (1) are 

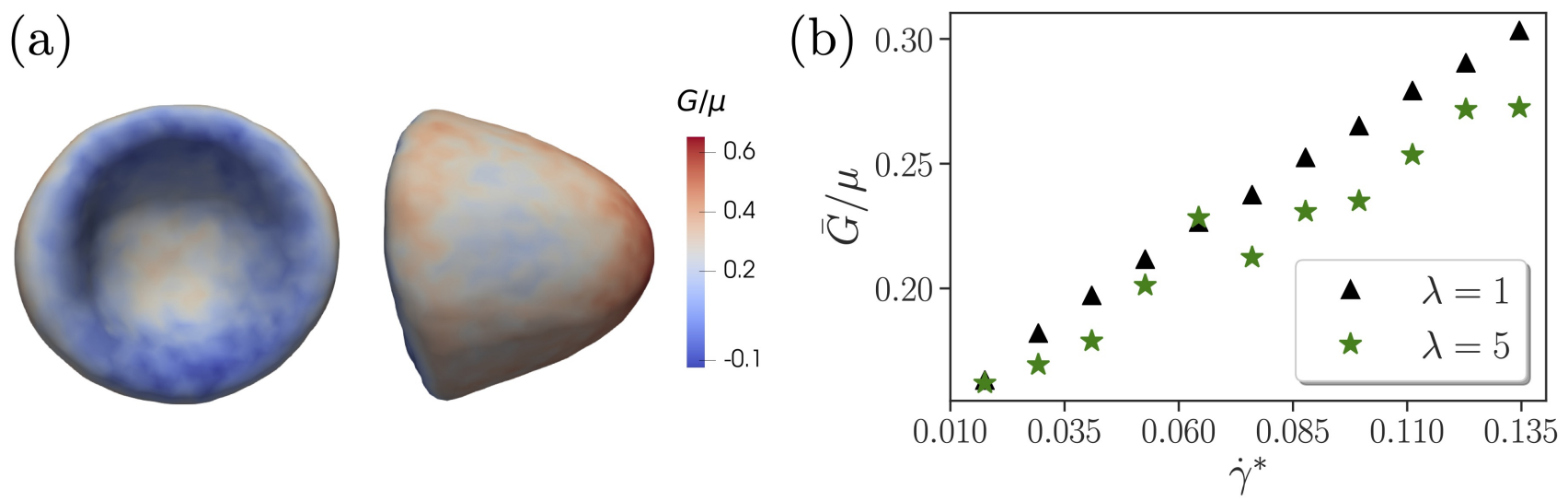

Figure 4. Membrane tension. (a) Side and front views of the parachute shape with a local tension $G$ indicated by the color code and normalized by the shear modulus $\mu$. Here, $\lambda=5, \chi=0.71$, and $\dot{\gamma}^{*}=0.076$. (b) Average tension $\bar{G} / \mu$ of the whole RBC as a function of non-dimensional shear rate $\dot{\gamma}^{*}$. Here, the confinement is fixed at $\chi=0.53$. The data are shown for two different viscosity contrasts $\lambda=1$ and $\lambda=5$. A jump in tension for $\lambda=5$ at $\dot{\gamma}^{*} \simeq 0.065$ corresponds to the tank-treading-to-parachute transition.

considered, even though the in-plane elastic-energy term supplies the maximum contribution to $G$. The concave part of the parachute shape has a significantly lower tension than the convex front of the RBC exposed to strong fluid stresses. The tension distribution for tumbling and tank-treading RBCs has a qualitatively similar trend, in which the frontal part of the cell has larger tension than the back portion. However, for tumbling and tank-treading states, local tension fluctuates in accord with the discussed RBC dynamics, while for the parachute state, temporal tension changes are generally small.

Figure $4(\mathrm{~b})$ presents tension $\bar{G}$ averaged over the RBC surface as a function of $\dot{\gamma}^{*}$ for two different viscosity contrasts $\lambda=1$ and $\lambda=5$. The average tension increases with the shear rate $\dot{\gamma}^{*}$ in an almost linear fashion for both viscosity contrasts. Interestingly, $\lambda=5$ generally leads to a lower tension in comparison with $\lambda=1$, which is consistent with a previous numerical investigation [60] showing that the maximum tension increases with the flow rate and decreases with increasing viscosity contrast. For $\lambda=5$, there is a jump in tension at approximately $\dot{\gamma}^{*} \simeq 0.065$ that corresponds to the tank-treading-to-parachute transition as shown in Fig. 1. Note that such jump is not present for $\lambda=1$.

An average tension of about $\bar{G}=10^{-6} \mathrm{~N} / \mathrm{m}$ (or $\left.\bar{G} / \mu \approx 0.2\right)$ is comparatively large. For example, in a recent study on sculpting of lipid vesicles by enclosed active particles [61], complex vesicle shapes have been observed for floppy vesicles with a tension of about $10^{-8} \mathrm{~N} / \mathrm{m}$, while a high membrane tension of about $10^{-5} \mathrm{~N} / \mathrm{m}$ completely suppresses any vesicle shape changes. Apart from the average tension, it is also instructive to look at the maximum tension $G_{\max }=\max \left\{G_{i}\right\}$ for different $\chi$ and $\dot{\gamma}^{*}$. As expected, $G_{\max }$ increases with increasing shear rate. For $\lambda=5$, the maximum tension at $\chi=0.62$ is $\mathrm{G}_{\max } / \mu=0.7$ for $\dot{\gamma}^{*}=0.053$ and $\mathrm{G}_{\max } / \mu=0.77$ for $\dot{\gamma}^{*}=0.076$ (both are parachute states). For a given shear rate, an increase in confinement results in elevation of $G_{\max }$, e.g. for $\dot{\gamma}^{*}=0.053, \mathrm{G}_{\max } / \mu=0.64$ for $\chi=0.35$ (tumbling state) and $\mathrm{G}_{\max } / \mu=0.7$ for $\chi=0.62$ (parachute state). These trends are similar for $\lambda=1$. However, differences in $G_{\max }$ with respect to the viscosity contrast are rather small, indicating that external fluid stresses mainly govern the membrane tension. The magnitudes of maximal tension from our simulations are consistent with the values reported in Ref. [60]. 
bioRxiv preprint doi: https://doi.org/10.1101/2021.02.11.430779; this version posted February 11, 2021. The copyright holder for this preprint (which was not certified by peer review) is the author/funder, who has granted bioRxiv a license to display the preprint in perpetuity. It is made available under aCC-BY-NC 4.0 International license.

\section{DISCUSSION AND CONCLUSIONS}

In our study, we have focused on the effect of viscosity contrast $\lambda$ on RBC dynamic states in microcapillary flow. State diagrams with different dynamic states, such as snaking, tumbling, tank-treading, and parachute, have been constructed for several $\lambda$ values and wide ranges of non-dimensional shear rates $\dot{\gamma}^{*}$ and confinements $\chi$. Our central result is that there are significant changes in the state diagram when the viscosity contrast is decreased from $\lambda=5$ to $\lambda=1$. In particular, the region of stable parachutes becomes larger and expands toward lower confinements with decreasing $\lambda$. This result seems to be in contradiction to the fact that a large viscosity inside the RBC dampens membrane dynamics and hence, should suppress the dynamic tank-treading state [39, 40]. To verify the robustness of our simulation predictions, we have performed simulations with a consecutive change in the viscosity contrast for several conditions where parachutes are stable for $\lambda=1$ and tank-treading is stable for $\lambda=5$. Thus, after reaching a stable parachute state for $\lambda=1$, the viscosity contrast is instantaneously switched to $\lambda=5$, leading to the tank-treading state. Then, switching back to $\lambda=1$ brings the initially tank-treading RBC to the parachute state. Furthermore, a larger parachute region for $\lambda=1$ than that for $\lambda=5$ has also been observed in 2D simulations of vesicles [24, 25].

To reconcile this seeming contradiction, physical mechanisms that govern the parachute-to-tank-treading transition in tube flow have to be uncovered. A study based on 2D simulations of vesicles [23] suggests that the parachute-to-slipper transition can be described well by a pitchfork bifurcation and that a slipper shape provides a higher flow efficiency for RBCs. Unfortunately, this argument has not been connected in any way to the viscosity contrast or internal cell dissipation. From existing experimental and simulation studies [15, 17, 20, 24, 25, 29, 34], it is clear that the parachute state requires large enough flow rates, such that flow stresses in the tube center are sufficient to deform the RBC into a parachute shape. Therefore, only when a RBC conforms well enough to the flow profile, the parachute state is stable. Note that we have not found substantial differences in parachute shapes for different viscosity contrasts, which is likely due to the fact that a non-dynamic parachute state of the RBC depends primarily on its elastic properties, and is nearly independent of internal dissipation. Our hypothesis is that membrane dynamics is important for parachute stability, in the following sense. As the parachute-to-tank-treading transition is approached, a perturbation (e.g., due to cell diffusion) in RBC position from the tube center leads to asymmetry in fluid-flow stresses which pull the RBC away from the center and set the membrane into a tank-treading-like motion. A slight motion of the membrane in the parachute state is observed in our simulations, as the $\mathrm{RBC}$ is never perfectly symmetric and is often located slightly away from the tube center. For $\lambda=1$, the membrane can rotate faster than in case of $\lambda=5$, and therefore, the mismatch between local membrane motion and fluid flow is smaller, resulting in reduced local fluid stresses that pull the RBC away from the center. For $\lambda=5$, the local fluid stresses on the RBC are larger due to slow membrane tank-treading, leading to the destabilization of parachute shape at larger confinements in comparison to $\lambda=1$.

Another important difference in the state diagrams for $\lambda=1$ and $\lambda=5$ is that the tumbling-to-tanktreading transition occurs at larger shear rates for $\lambda=5$ than for $\lambda=1$. This can be explained by the fact that an increased dissipation inside the $\mathrm{RBC}$ for $\lambda=5$ suppresses tank-treading motion and delays the transition in terms of $\dot{\gamma}^{*}$. In fact, in simple shear flow, the tank-treading state does not exist for $\lambda=5$ [39, 40]. For microcapillary flow, RBC tank-treading becomes possible at $\lambda=5$ due to the confinement which can trigger the tumbling-to-tank-treading transition even when cell dimensions are smaller than the distance between two walls [62]. For a large enough vessel diameter, it is plausible to expect that the tank-treading state should disappear for $\lambda=5$, as local flow conditions should closely resemble simple shear flow at the scale of RBC size. For instance, recent microfluidic experiments in a square channel [34] 
have reported the existence of rotating trilobe shapes at low confinements and high flow rates, which are consistent with RBC shapes in simple shear flow at $\lambda=5$ [39, 40].

Membrane tension must be directly related to mechano-transduction as RBC membrane contains many mechano-sensitive channels [58, 59]. We have shown that an increase in the viscosity contrast lowers the membrane tension. A high viscosity of the cytosol provides a large dissipation, reducing membrane tension. Furthermore, the maximum tension increases with increasing shear rate $\dot{\gamma}^{*}$ and confinement $\chi$. Several experimental studies show that flow stresses can change RBC biochemical properties. For instance, when RBCs pass through small constrictions, they release ATP which can participate in vasodilation signaling [63, 64]. Furthermore, a recent investigation [65] reports that when RBCs pass through small constrictions, the mechano-sensitive channels (e.g., Piezo1 and Gardos channels) that participate in RBC volume control become activated. The relevance of membrane tension has also been demonstrated for malaria disease, such that an increased RBC membrane tension in Dantu blood group significantly reduces the invasion of RBCs by malaria parasites, which is a protective mechanism from malaria infection [66].

\section{CONFLICT OF INTEREST STATEMENT}

The authors declare no conflicts of interest.

\section{AUTHOR CONTRIBUTIONS}

A.K.D and J.M. performed simulations and analyzed the data. G.G. and D.A.F. designed research. D.A.F. supervised the project. All authors discussed the results and wrote the manuscript.

\section{REFERENCES}

[1] Popel AS, Johnson PC. Microcirculation and hemorheology. Annu. Rev. Fluid Mech. 37 (2005) $43-69$.

[2] Lipowsky HH. Microvascular rheology and hemodynamics. Microcirculation 12 (2005) 5-15.

[3] Pries AR, Secomb TW. Blood flow in microvascular networks. Tuma RF, Duran WN, Ley K, editors, Handbook of Physiology, The Cardiovascular System, Microcirculation (San Diego: Academic Press) (2008), 3-36.

[4] Secomb TW. Blood flow in the microcirculation. Annu. Rev. Fluid Mech. 49 (2017) 443-461.

[5] Gompper G, Fedosov DA. Modeling microcirculatory blood flow: current state and future perspectives. WIREs Syst. Biol. Med. 8 (2016) 157-168.

[6] Evans EA, Skalak R. Mechanics and thermodynamics of biomembranes (Boca Raton, Florida: CRC Press, Inc.) (1980).

[7] Discher DE, Mohandas N, Evans EA. Molecular maps of red cell deformation: hidden elasticity and in situ connectivity. Science 266 (1994) 1032-1035.

[8] Diez-Silva M, Dao M, Han J, Lim CT, Suresh S. Shape and biomechanical characteristics of human red blood cells in health and disease. MRS Bulletin 35 (2010) 382-388.

[9] Tomaiuolo G. Biomechanical properties of red blood cells in health and disease towards microfluidics. Biomicrofluidics 8 (2014) 051501.

[10] Kaul DK, Fabry ME, Windisch P, Baez S, Nagel RL. Erythrocytes in sickle cell anemia are heterogeneous in their rheological and hemodynamic characteristics. J. Clin. Invest. 72 (1983) 22-31.

[11] Cranston HA, Boylan CW, Carroll GL, Sutera SP, Williamson JR, Gluzman IY, et al. Plasmodium falciparum maturation abolishes physiologic red cell deformability. Science 223 (1984) 400-403. 
bioRxiv preprint doi: https://doi.org/10.1101/2021.02.11.430779; this version posted February 11, 2021. The copyright holder for this preprint (which was not certified by peer review) is the author/funder, who has granted bioRxiv a license to display the preprint in perpetuity. It is made available under aCC-BY-NC 4.0 International license.

[12] Fedosov DA, Caswell B, Suresh S, Karniadakis GE. Quantifying the biophysical characteristics of Plasmodium-falciparum-parasitized red blood cells in microcirculation. Proc. Natl. Acad. Sci. USA 108 (2011) 35-39.

[13] Skalak R, Branemark PI. Deformation of red blood cells in capillaries. Science 164 (1969) 717-719.

[14] Gaehtgens P, Dührssen C, Albrecht KH. Motion, deformation, and interaction of blood cells and plasma during flow through narrow capillary tubes. Blood Cells 6 (1980) 799-812.

[15] Bagge U, Branemark PI, Karlsson R, Skalak R. Three-dimensional observations of red blood cell deformation in capillaries. Blood Cells 6 (1980) 231-237.

[16] Tomaiuolo G, Preziosi V, Simeone M, Guido S, Ciancia R, Martinelli V, et al. A methodology to study the deformability of red blood cells flowing in microcapillaries in vitro. Ann Ist Super Sanita $\mathbf{4 3}$ (2007) 186-192.

[17] Tomaiuolo G, Simeone M, Martinelli V, Rotoli B, Guido S. Red blood cell deformation in microconfined flow. Soft Matter 5 (2009) 3736-3740.

[18] Guido S, Tomaiuolo G. Microconfined flow behavior of red blood cells in vitro. C. R. Physique $\mathbf{1 0}$ (2009) 751-763.

[19] Abkarian M, Faivre M, Stone HA. High-speed microfluidic differential manometer for cellular-scale hydrodynamics. Proc. Natl. Acad. Sci. USA 103 (2006) 538-542.

[20] Abkarian M, Faivre M, Horton R, Smistrup K, Best-Popescu CA, Stone HA. Cellular-scale hydrodynamics. Biomed. Mater. 3 (2008) 034011.

[21] Secomb TW, Skalak R, Özkaya N, Gross JF. Flow of axisymmetric red blood cells in narrow capillaries. J. Fluid Mech. 163 (1986) 405-423.

[22] Secomb TW, Styp-Rekowska B, Pries AR. Two-dimensional simulation of red blood cell deformation and lateral migration in microvessels. Ann. Biomed. Eng. 35 (2007) 755-765.

[23] Kaoui B, Biros G, Misbah C. Why do red blood cells have asymmetric shapes even in a symmetric flow? Phys. Rev. Lett. 103 (2009) 188101.

[24] Kaoui B, Tahiri N, Biben T, Ez-Zahraouy H, Benyoussef A, Biros G, et al. Complexity of vesicle microcirculation. Phys. Rev. E 84 (2011) 041906.

[25] Tahiri N, Biben T, Ez-Zahraouy H, Benyoussef A, Misbah C. On the problem of slipper shapes of red blood cells in the microvasculature. Microvasc. Res. 85 (2013) 40-45.

[26] Aouane O, Thiébaud M, Benyoussef A, Wagner C, Misbah C. Vesicle dynamics in a confined Poiseuille flow: from steady state to chaos. Phys. Rev. E 90 (2014) 033011.

[27] Lázaro GR, Hernández-Machado A, Pagonabarraga I. Rheology of red blood cells under flow in highly confined microchannels: I. effect of elasticity. Soft Matter 10 (2014) 7195-7206.

[28] Noguchi H, Gompper G. Shape transitions of fluid vesicles and red blood cells in capillary flows. Proc. Natl. Acad. Sci. USA 102 (2005) 14159-14164.

[29] Fedosov DA, Peltomäki M, Gompper G. Deformation and dynamics of red blood cells in flow through cylindrical microchannels. Soft Matter 10 (2014) 4258-4267.

[30] Ye T, Phan-Thien N, Khoo BC, Lim CT. Dissipative particle dynamics simulations of deformation and aggregation of healthy and diseased red blood cells in a tube flow. Phys. Fluids 26 (2014) 111902.

[31] Fedosov DA, Noguchi H, Gompper G. Multiscale modeling of blood flow: from single cells to blood rheology. Biomech. Model. Mechanobiol. 13 (2014) 239-258.

[32] Ye T, Shi H, Peng L, Li Y. Numerical studies of a red blood cell in rectangular microchannels. J. Appl. Phys. 122 (2017) 084701.

[33] Guckenberger A, Kihm A, John T, Wagner C, Gekle S. Numerical-experimental observation of shape bistability of red blood cells flowing in a microchannel. Soft Matter 14 (2018) 2032-2043. 
[34] Reichel F, Mauer J, Nawaz AA, Gompper G, Guck J, Fedosov DA. High-throughput microfluidic characterization of erythrocyte shapes and mechanical variability. Biophys. J. 117 (2019) 14-24.

[35] Fischer TM. Shape memory of human red blood cells. Biophys. J. 86 (2004) 3304-3313.

[36] Skotheim JM, Secomb TW. Red blood cells and other nonspherical capsules in shear flow: oscillatory dynamics and the tank-treading-to-tumbling transition. Phys. Rev. Lett. 98 (2007) 078301.

[37] Abkarian M, Faivre M, Viallat A. Swinging of red blood cells under shear flow. Phys. Rev. Lett. 98 (2007) 188302.

[38] Dupire J, Socol M, Viallat A. Full dynamics of a red blood cell in shear flow. Proc. Natl. Acad. Sci. USA 109 (2012) 20808-20813.

[39] Lanotte L, Mauer J, Mendez S, Fedosov DA, Fromental JM, Claveria V, et al. Red cells' dynamic morphologies govern blood shear thinning under microcirculatory flow conditions. Proc. Natl. Acad. Sci. USA 113 (2016) 13289-13294.

[40] Mauer J, Mendez S, Lanotte L, Nicoud F, Abkarian M, Gompper G, et al. Flow-induced transitions of red blood cell shapes under shear. Phys. Rev. Lett. 121 (2018) 118103.

[41] Cokelet GR, Meiselman HJ. Rheological comparison of hemoglobin solutions and erythrocyte suspensions. Science 162 (1968) 275-277.

[42] Wells R, Schmid-Schönbein H. Red cell deformation and fluidity of concentrated cell suspensions. $J$. Appl. Physiol 27 (1969) 213-217.

[43] Yazdani AZK, Bagchi P. Phase diagram and breathing dynamics of a single red blood cell and a biconcave capsule in dilute shear flow. Phys. Rev. E 84 (2011) 026314.

[44] Sinha K, Graham MD. Dynamics of a single red blood cell in simple shear flow. Phys. Rev. E 92 (2015) 042710.

[45] Cordasco D, Yazdani A, Bagchi P. Comparison of erythrocyte dynamics in shear flow under different stress-free configurations. Phys. Fluids 26 (2014) 041902.

[46] Gompper G, Kroll DM. Triangulated-surface models of fluctuating membranes. Nelson DR, Piran T, Weinberg S, editors, Statistical mechanics of membranes and surfaces (Singapore: World Scientific). 2nd edn. (2004), 359-426.

[47] Fedosov DA, Caswell B, Karniadakis GE. A multiscale red blood cell model with accurate mechanics, rheology, and dynamics. Biophys. J. 98 (2010) 2215-2225.

[48] Fedosov DA, Caswell B, Karniadakis GE. Systematic coarse-graining of spectrin-level red blood cell models. Comput. Meth. Appl. Mech. Eng. 199 (2010) 1937-1948.

[49] Español P, Revenga M. Smoothed dissipative particle dynamics. Phys. Rev. E 67 (2003) 026705.

[50] Müller K, Fedosov DA, Gompper G. Smoothed dissipative particle dynamics with angular momentum conservation. J. Comp. Phys. 281 (2015) 301-315.

[51] Fedosov DA, Karniadakis GE. Triple-decker: Interfacing atomistic-mesoscopic-continuum flow regimes. J. Comp. Phys. 228 (2009) 1157-1171.

[52] Evans EA. Bending elastic modulus of red blood cell membrane derived from buckling instability in micropipet aspiration tests. Biophys. J. 43 (1983) 27-30.

[53] Dao M, Lim CT, Suresh S. Mechanics of the human red blood cell deformed by optical tweezers. $J$. Mech. Phys. Solids 51 (2003) 2259-2280.

[54] Yoon YZ, Kotar J, Yoon G, Cicuta P. The nonlinear mechanical response of the red blood cell. Phys. Biol. 5 (2008) 036007.

[55] Goldsmith HL, Marlow J. Flow behaviour of erythrocytes. I. Rotation and deformation in dilute suspensions. Proc. R. Soc. Lond. B 182 (1972) 351-384. 
[56] Fischer TM, Stöhr-Liesen M, Schmid-Schönbein H. The red cell as a fluid droplet: tank tread-like motion of the human erythrocyte membrane in shear flow. Science 202 (1978) 894-896.

[57] Dasanna AK, Fedosov DA, Gompper G, Schwarz US. State diagram for wall adhesion of red blood cells in shear flow: from crawling to flipping. Soft Matter 15 (2019) 5511-5520.

[58] Coste B, Mathur J, Schmidt M, Earley TJ, Ranade S, Petrus MJ, et al. Piezo1 and Piezo2 are essential components of distinct mechanically activated cation channels. Science 330 (2010) 55-60.

[59] Zarychanski R, Schulz VP, Houston BL, Maksimova Y, Houston DS, Smith B, et al. Mutations in the mechanotransduction protein PIEZO1 are associated with hereditary xerocytosis. Blood $\mathbf{1 2 0}$ (2012) 1908-1915.

[60] Omori T, Ishikawa T, Barthés-Biesel D, Salsac AV, Imai Y, Yamaguchi T. Tension of red blood cell membrane in simple shear flow. Phys. Rev. E 86 (2012) 056321.

[61] Vutukuri HR, Hoore M, Abaurrea-Velasco C, van Buren L, Dutto A, Auth T, et al. Active particles induce large shape deformations in giant lipid vesicles. Nature 586 (2020) 52-56.

[62] Kaoui B, Krüger T, Harting J. How does confinement affect the dynamics of viscous vesicles and red blood cells? Soft Matter 8 (2012) 9246-9252.

[63] Wan J, Ristenpart WD, Stone HA. Dynamics of shear-induced ATP release from red blood cells. Proc. Natl. Acad. Sci. USA 105 (2008) 16432-16437.

[64] Forsyth AM, Wan J, Owrutsky PD, Abkarian M, Stone HA. Multiscale approach to link red blood cell dynamics, shear viscosity, and ATP release. Proc. Natl. Acad. Sci. USA 108 (2011) 10986-10991.

[65] Danielczok JG, Terriac E, Hertz L, Petkova-Kirova P, Lautenschläger F, Laschke MW, et al. Red blood cell passage of small capillaries is associated with transient $\mathrm{Ca}^{2+}$-mediated adaptations. Front. Physiol. 8 (2017) 979.

[66] Kariuki SN, Marin-Menendez A, Introini V, Ravenhill BJ, Lin YC, Macharia A, et al. Red blood cell tension protects against severe malaria in the Dantu blood group. Nature 585 (2020) 579-583. 